\title{
ESTUDO DAS CONDIÇÕES DE SAÚDE DAS CRIANÇAS DO MUNICÍPIO DE LONDRINA,
}

PR, 1990.

\author{
JOÄO JOSÉ BATISTA DE CAMPOS ' \\ ALDAISA CASSANHO FORSTER 2 \\ DARLI ANTÓNIO SOARES 3
}

\begin{abstract}
CAMPOS, João José Batista de; FORSTER, Aldaisa Cassanho; SOARES, Darli Antônio. Estudo das condiçōes de saúde das crianças do Município de Londrina. Semina: Ci. Biol./Saúde, Londrina, v. 16, n. 2, p. 224-233, jun. 1995 .
\end{abstract}

\begin{abstract}
RESUMO: Visando contribuir para a avaliaçáo dos serviços de saúde matemo-infantil em Londrina, PR, foi realizada esta investigaçăo, que teve como objetivo estudar as condiçőes de assisténcia básica a crianças da zona urbana do municipio de Londrina, em 1990. Este tipo de pesquisa, do ponto de vista metodológico, é denominada de estudo transversat. A população de estudo correspondeu a uma amostra populacional de 388 crianças menores de 5 anos, das cinco regiōes urbanas da cidade. As variáveis abordadas foram: escolaridade do chefe de familia, renda percapita e classe social. Na caracterizaçăo socio-econômica, destacou-se o alto grau de escolaridade dos chefes de familia, guardando uma relaçăo direta com a renda per-capita, e associaçåo significativa em relaçăo à classe social. Na análise do pré-natal de 383 gestantes em $82 \%$ dos casos, houve referéncia a 6 ou mais consultas; em relaçăo ao parto $55,8 \%$ foram cirúrgicos; $79,4 \%$ fizeram puericultura; em relaçăo ao número de consultas no primeiro ano de vida a média foi de 10,8 consultas por criança; a vacinaçăo mostrou uma cobertura elevada quanto ao esquema básico: 98\% foram imunizadas com uma dose da vacina BCG, 98\% receberam as tres doses da Sabin, $98 \%$ as tres doses de DPT e $98 \%$ a Anti-Sarampo. Os resultados obtidos levam à conclusăo de que a assisténcia materno-infantil prestada à populaçăo foi ampla, năo sendo evidenciada desigualdade significativa, quanto aos diferentes estratos sociais.
\end{abstract}

PALAVRAS-CHAVE: Saúde da Criança, Avaliaçăo de Serviços de Saúde, Assistência Matemo infantil.

\section{1 - INTRODUÇĀO}

A análise das condições de saúde da população é importante frente à reorganização dos serviços de saúde, colaborando para as práticas de planejamento, administração e de avaliação crítica e contínua de sua orientação; como também, contribuindo para os movimentos e reivindicações populares.

Nesta perspectiva, em Londrina, Paraná, é importante conhecer a evolução da atenção primária à saúde, que teve o seu início na década de $70 \mathrm{com}$ a estruturação do curso de medicina, trazendo para o norte do Paraná, professores com formaçăo e experiência na área de Saúde Coletiva. Esse processo se desenvolveu com ritmos diferenciados, em função das conjunturas municipal e estadual (ALMEIDA, 1979).

No início da década de 80 , com a intensificação da expansão da rede de serviços básicos, através de um plano de saúde do governo estadual (CORDONI, 1987), coincidente com o plano de saúde municipal, houve implementação destas políticas em Londrina, que se ampliaram ainda mais com a reorientação da assistência médica da Previdência Social, particularmente pelas Ações Integradas de Saúde, e posteriormente pelo Sistema Unificado e Descentralizado de Saúde, como importante reforço à estruturação do Sistema Único de Saúde (UNIVERSIDADE ESTADUAL DE LONDRINA, 1992).

Destaca-se a evolução dos principais indicadores de saúde, como o decréscimo do coeficiente de mortalidade infantil e das doenças de notificação compulsória, sendo essas variaçốes possivelmente relacionadas com o impacto de determinadas açōes de saúde. Devido às limitações das fontes de informação, deve-se utilizar de forma cautelosa o sistema oficial de registro, como sugerem BARROS \& VICTORA, 1991.

Através do sistema de informaçōes sobre mortalidade e morbidade da Secretaria Estadual de Saúde, considerando os valores do coeficiente de mortalidade infantil por mil nascidos vivos para os anos de 1979 a 1990 , verificou-se que houve uma redução de 37 para 21 com tendência de queda durante o período. A redução da mortalidade proporcional de menores de 1 ano, para o mesmo período foi de $19,1 \%$ para $8,7 \%$. O declínio do número de doenças de notificação compulsória, pode ser constatado pela eliminação de

1 - Depto Materno Infantil e Saúde Comunitária/CCSJUniversidade Estadual de Londrina, Caixa Postal 1562, Londrina, PR, Brasil, CEP $86038-440$.

2 - Depto Medicina Socialfaculdade de Medicina de Ribeirăo Preto/Universidade de Såo Paulo, Campus Universitário - USP, Ribeirâo Preto, SP. Brasil, CEP $14049-900$.

3 - Deplo Materno Infantil e Saúde Comunitária/CCSrUniversidade Estadual de Londrina, Caixa Postal 1562, Londrina, PR, Brasil, CEP $86038-440$. 
casos de tétano neonatal; não houve casos de difteria registrados de 1987 a 1992; o último óbito por coqueluche ocorreu em 1989; no sarampo observou-se menor número de casos entre menores de cinco anos e a perda da sazonalidade; a meningite por tuberculose está praticamente controlada e a erradicação da poliomielite é uma realidade (Seção de Epidemiologia da $17^{\mathrm{a}}$ Regional SUS/Londrina).

Com o objetivo de estudar as ações de atenção primária à saúde matemo-infantil, desenvolvidas na cidade de Londrina, estudou-se a assistência de pré-natal, ao parto, puericultura e vacinação, relacionando estas variáveis com a estrutura social.

\section{2 - MATERIAL E MÉTODO}

Londrina, cidade que completou 57 anos em 1992, situa-se no norte do Estado do Paraná, com 387.913 habitantes, segundo dados preliminares do Censo 91 , concentrando $94 \%$ dessa população na área urbana, onde foi realizado o estudo (IBGE, 92).

A cidade dispõe de serviços de assistência à saúde que incluem 50 Postos de Saúde, dois Centros de Saúde, um Centro Integrado de Apoio Diagnóstico, uma Maternidade inaugurada no final de 92 e um Hospital na zona rural, gerenciados pelo município; um Centro Regional de Especialidades, dois Hospitais Gerais e um Hemocentro público administrados pela Secretaria Estadual de Saúde; um Hospital Geral Universitário e um Centro Odontológico vinculados à Universidade Estadual de Londrina (UEL).

Londrina dispõe também de rede de assistência filantrópica, com dois Hospitais Gerais de alta complexidade, um Ambulatório de Especialidades, uma Clínica Psiquiátrica que mantém tanto ala de ambulatório como de internamento, além de um Hospital de Referência Estadual para Oncologia e um Hospital especializado em assistência à saúde da mulher.

Compỏem ainda a rede assistencial do Sistema Único de Saúde, os seguintes recursos contratados: 219 médicos, 24 dentistas, 6 clínicas de fisioterapia, 8 laboratórios de análises clínicas, 5 hospitais privados, sendo 1 ortopédico, 1 infantil, 2 psiquiátricos e 1 matemoinfantil (UNIVERSIDADE ESTADUAL DE LONDRINA, 1992).

O número de domicilios a serem sorteados foi feito com o objetivo de analisar aproximadamente 1.000 crianças. Estas representavam $2,2 \%$ das 44.937 crianças menores de cinco anos de Londrina em 1989. Além disso, decidiu-se que seria incluída apenas uma criança por domicilio, para que as regiōes da cidade com maior número de crianças não ficassem superrepresentadas na amostra, o que por outro lado impediu que se alcançasse a meta numérica. O cadastro de domicílios utilizado foi o da Companhia Paranaense de Energia Elétrica, que abastece a toda área urbana de Londrina e é considerado um serviço de infra-estrutura de qualidade.

O método de amostragem foi sistemático, uma vez que os domicilios que participam da amostra foram determinados a partir de intervalos fixos. A quota de domicilios de cada bairro da cidade, foi escolhida em função de seu número de residências. O questionário utilizado tinha 7 partes, num total de 42 itens (Figura 1). A coleta de dados da pesquisa foi efetuada entre julho e dezembro de 1990, desenvolvida conforme a metodologia dos "inquéritos domiciliares" (MOSER, 1958).

Foram estudadas as variáveis citadas por vários autores (CARVALHEIRO, 1975; FORSTER, 1979; BARROS, 1983; ITO, 1984; VICTORA, 1988; MONTEIRO, 1988), como tendo associação importante com as condições de saúde materno-infantil: renda per capita, escolaridade do chefe de família e classes sociais. Do chefe de familia, os dados coletados foram a idade, escolaridade, ocupação principal, posição na ocupação e renda; tendo sido aplicada a proposta de operacionalização de classes, desenvolvida por BARROS (1983) e adaptada por FORSTER (1984).

$\mathrm{Na}$ análise da assistência à saúde da mãe e da criança foram selecionadas as quatro variáveis mais relevantes na caracterização da cobertura dos serviços de saúde na zona urbana do Município de Londrina: prénatal, parto, puericultura e vacinação. $\mathrm{Na}$ análise do esquema de imunizações, foi considerada apenas a informação fornecida pela carteira de vacina. A criança foi considerada imunizada caso tivesse tomado uma dose de BCG intradérmico, as três doses da Vacina Sabin, três doses da DPT e uma dose da Anti-Sarampo, portanto, foram incluidas apenas as crianças com mais de doze meses de idade.

Para descrever o domicílio em que viviam as crianças, obteve-se o número de cômodos, número de pessoas por cômodo de dormir e condições de saneamento básico das familias entrevistadas.

Os dados obtidos a partir das entrevistas foram convertidos em arquivos computadorizados, após análise de consistência, a base de dados foi lida pelo programa de análise estatistica SAS (Statistic Analysis Sistem) versão 5.0 .

\section{3 - RESULTADOS}

A amostra estudada correspondeu a 388 crianças menores de cinco anos. O fluxograma de obtenção da amostra se encontra esquematizado na Figura 2.

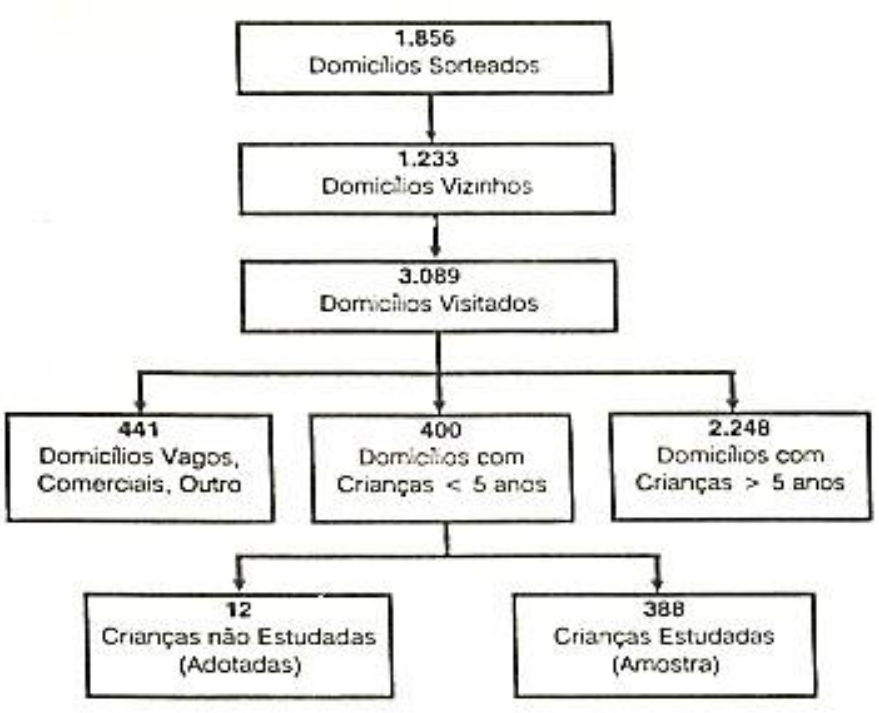

Figura 2 - Fluxograma do processo de amostragem 
A đuração média da entrevista foi de 16 minutos. Quanto à idade das crianças estudadas observou-se que as menores de 12 meses (93) representaram menos que $25 \%$ da amostra. Com relação à idade dos pais, verificou-se que a maioria tinham entre 20 e 40 anos, 354 măes $(91 \%)$ e 310 pais $(80 \%)$.

Em torno de $46 \%$ das residências apresentavam dois cômodos, considerando apenas cômodo de dormir, e $20 \%$ apenas um cômodo. Com relação ao número médio de pessoas por domicilio, foi obtido 4,5 . Observouse que $37 \%$ das familias possuem 5 ou mais pessoas.

Quanto ao saneamento ambiental das casas, $99,2 \%$ das moradias estavam ligadas à rede pública de água e contavam com canalização interna. Já em relaçăo ao esgoto, apenas $40,5 \%$ dos domicilios estavam ligados à rede pública, ficando o restante na dependência de fossas. Para completar a descrição do saneamento. obteve-se a informação de que $99,7 \%$ das crianças residiam em domicilios servidos regularmente pela coleta pública do lixo.

\section{1 - Caracterização das famílias \\ A distribuição das crianças segundo o rendimento}

médio mensal das familias entrevistadas, mostrou que em tomo de $50 \%$ das crianças pertenciam a familias com rendimento inferiores a 5 salários minimos mensais; enquanto que aproximadamente $18 \%$ das familias possuiam renda igual ou superior a 10 salários mínimos. Em relaçåo ao rendimento médio mensal das mães, apenas $4 \%$ eram responsáveis pela sustentação financeira da criança e $67 \%$ delas năo tinham rendimentos. A renda em salário mínimo mensal percapita das familias estudadas, evidenciou que $76 \%$ delas percebiam entre 0 e 2 salários minimos.

Nåo foi encontrada diferença estatisticamente significativa, na análise entre os niveis de escolaridade alcançados pela mãe e pelo chefe de familia, sendo observada baixa proporção de analfabetismo 13 (3,3\%). Completaram o primário proporção ligeiramente superior dos chefes de familia $67(17,3 \%)$. Chamou atençăo o grande percentual de chefes de familia que chegaram ao ginasial $139(35,8 \%)$; enquanto no superior apenas $44(11,4 \%)$.

Relacionando a renda per capita à escolaridade do chefe de familia, observou-se associação estatisticamente significativa, Gráfico 1.

\section{GRÁFICO 1 - DISTRIBUIÇÃO DOS CHEFES DE FAMILIA SEGUNDO ASUA ESCOLARIDADE E OS DIFERENTES NIVEIS DE RENDA PER CAPITA, LONDRINA, 1990}

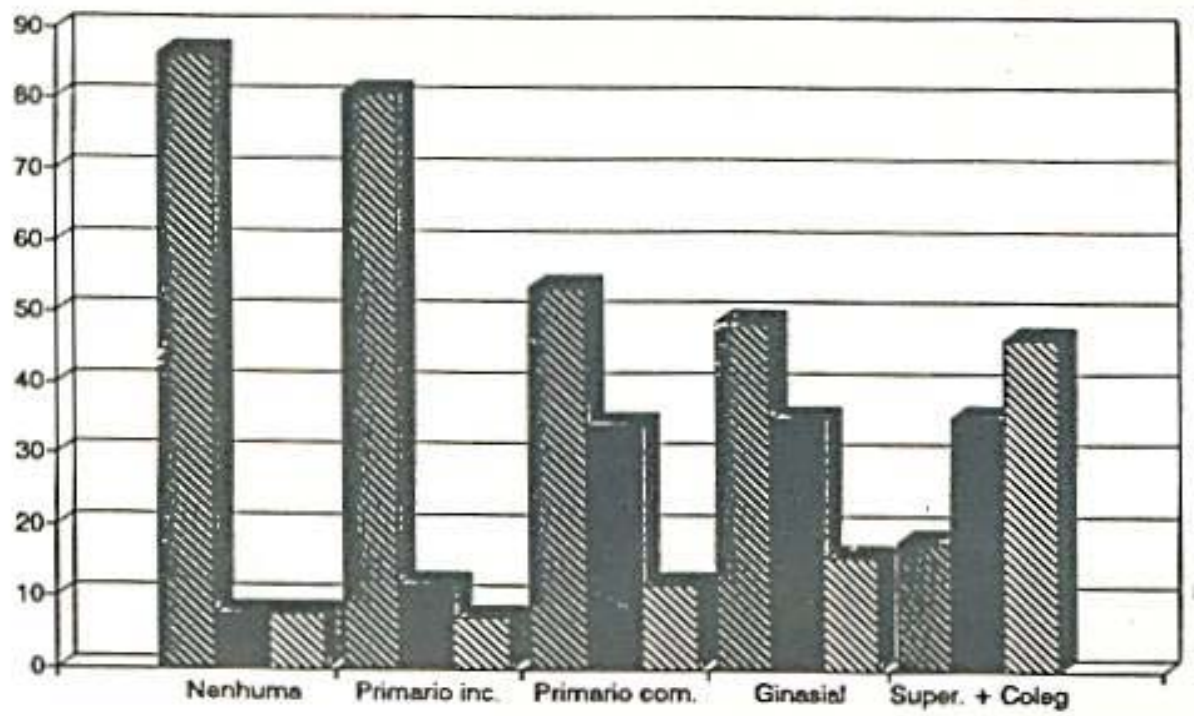

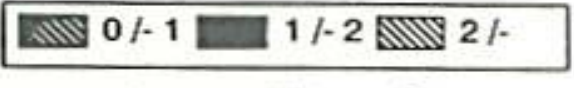

renda em salárlos mínimos
Para estudar a utilização dos serviços de saúde segundo as classes sociais e frações, considerou-se como parâmetro principalmente a inserçăo econômicosocial do chefe de familia.

O proletariado propriamente dito foi a classe majoritánia (65\%), a pequena burguesia representou cerca de $25 \%$ das crianças da cidade, à burguesia pertenciam em tomo de $6 \%$ das crianças e ao sub-proletariado menos de $3 \%$ delas e apenas $0,3 \%$ foi classificada como sendo sem classe.

\section{2 - Assistência à saúde materno-infantil}

\subsection{1 - Assistência pré-natal}

A concentraçăo alcançada na cidade de Londrina pela assistência pré-natal da gestante correspondente à criança selecionada, foi de 6 ou mais consultas para $82 \%$ dos casos; verificou-se também que a assistência foi iniciada no primeiro trimestre da gestação em $82 \%$ das mães, sendo apenas de $1,6 \%$ as măes que năo fizeram pré-natal.

Para verificar a influência das variáveis: renda per capita, escolaridade do chefe de familia e classe social, sobre a precocidade do pré-natal das mảes, foi utilizado o teste do $X^{2}$ e eliminando a coluna daquelas que năo 0 fizeram, e aplicando a correçăo de Yates. Observou-se que năo houve diferença estatisticamente significativa. Em todas as faixas ocorreu uma concentração no primeiro trimestre, diminuindo proporcionalmente a representaçăo de cada nível no total da amostra (Tabela 1). 


\section{Tabela 1 - DISTRIBUIÇÃO DAS FAMÍLIAS ESTUDADAS NA ZONA URBANA DO MUNICÍPIO DE LONDRINA, PELO INICIO DO PRÉ-NATAL E A RENDA PER CAPITA, 1990}

\begin{tabular}{|c|c|c|c|c|c|c|c|c|c|c|}
\hline \multirow{2}{*}{$\begin{array}{l}\text { Renda per-capita } \\
\text { (salário-mínimo) }\end{array}$} & \multicolumn{2}{|c|}{ Năo fez } & \multicolumn{2}{|l|}{$1^{\circ}$. tri } & \multicolumn{2}{|l|}{$2^{\circ} \cdot \operatorname{tri}$} & \multicolumn{2}{|l|}{$3^{\circ}$. tri } & \multicolumn{2}{|c|}{ TOTAL } \\
\hline & $\mathbf{N}$ & $\%$ & $N$ & $\%$ & & $\%$ & $\mathbf{N}$ & $\%$ & $\mathbf{N}$ & $\%$ \\
\hline $0-/ 1$ & 4 & 2,4 & 126 & 75,4 & 34 & 20,4 & 3 & 1,8 & 167 & 100,0 \\
\hline $1-12$ & 2 & 1,6 & 101 & 82,8 & 18 & 14,8 & 1 & 0,8 & 122 & 100,0 \\
\hline 2 - & 0 & 0,0 & 84 & 91,3 & 8 & 8,7 & 0 & 0,0 & 92 & 100,0 \\
\hline TOTAL & 6 & 1,6 & 311 & 81,6 & 60 & 15,7 & 4 & 1,1 & $381^{*}$ & 100,0 \\
\hline \multicolumn{11}{|c|}{ (*) Sete casos ignorados } \\
\hline $\begin{array}{l}X^{2} \text { calculado }=6,9 \\
X^{2} \text { critico }=15,50 \\
\left({ }^{*}\right)=\text { Nivel de sig }\end{array}$ & ** & $=5$ & & & & & & & & \\
\hline
\end{tabular}

\subsection{2 - Assistência ao parto}

Quanto à assistência ao parto, verificou-se que $85 \%$ das crianças nasceram em hospitais privados, e apenas $15 \%$ dos partos foram realizados no Hospital Universitário, o que demonstrou a baixa cobertura pública aos partos. Apesar desta realidade, a cobertura hospitalar foi praticamente universal, e identificou-se apenas um caso realizado fora do ambiente hospitalar.

Com relação à distribuição das modalidades de parto e considerando-se desfavoráveis e fruto de má assistência tanto proporções exiguas quanto proporções excessivas de partos cirúrgicos, pode-se observar que as cesarianas atingiram na amostra a freqüência inquestionavelmente excessiva de $55,8 \%$.

Analisando a distribuição dos partos de acordo com renda per capita, escolaridade do chefe de familia e classe social, pode ser observada diferença estatisticamente significativa, ou seja o parto cesariana esteve privilegiado entre as famílias de maior renda, melhor escolaridade e classe social superior (Tabelas 2, 3 e 4).

Tabela 2 - ESCOLARIDADE DO CHEFE DAS FAMILIAS ESTUDADAS NO MUNICÍPIO DE LONDRINA, SEGUNDO O TIPO DE PARTO, 1990

\begin{tabular}{|c|c|c|c|c|c|c|}
\hline \multirow{3}{*}{$\begin{array}{l}\text { Escolaridade } \\
\text { do chefe }\end{array}$} & \multicolumn{4}{|c|}{ Tipos de parto } & \multirow{2}{*}{\multicolumn{2}{|c|}{ TOTAL }} \\
\hline & \multicolumn{2}{|c|}{ Normal } & \multicolumn{2}{|c|}{ Cesárea } & & \\
\hline & $\mathbf{N}$ & $\%$ & $\mathbf{N}$ & $\%$ & $\mathbf{N}$ & $\%$ \\
\hline Nenhuma & 10 & 76,9 & 3 & 23.1 & 13 & 100,0 \\
\hline Primário inc. & 23 & 56,1 & 18 & 43,9 & 41 & 100,0 \\
\hline Primário com. & 30 & 44,8 & 37 & 55,2 & 67 & 100,0 \\
\hline Ginasial & 62 & 44,9 & 76 & 55,1 & 138 & 100,0 \\
\hline Super. + Coleg. & 45 & 35,7 & 81 & 64,3 & 126 & 100.0 \\
\hline TOTAL & 170 & 44,2 & 215 & 55,8 & $385^{\circ}$ & 100,0 \\
\hline
\end{tabular}

(*) Em três casos o parto foi à fórceps

$X^{2}$ calculado $=11,70^{* *}$

$X^{2}$ critico $=9,49$ (grau de liberdade $=4$ )

$\left({ }^{*}\right)=$ Nivel de significância $=5 \%$ 
Tabela 3 - DISTRIBUIÇAO DAS MULHERES SEGUNDO AS

CLASSES SOCIAIS E O TIPO DE PARTO, LONDRINA, 1990

\begin{tabular}{|c|c|c|c|c|c|c|}
\hline \multirow{3}{*}{$\begin{array}{l}\text { Classe } \\
\text { Social }\end{array}$} & \multicolumn{4}{|c|}{ Tipo de parto } & \multirow{2}{*}{\multicolumn{2}{|c|}{ TOTAL }} \\
\hline & \multicolumn{2}{|c|}{ Normal } & \multicolumn{2}{|c|}{ Cesárea } & & \\
\hline & $\mathrm{N}$ & $\%$ & $\mathbf{N}$ & $\%$ & $\mathbf{N}$ & $\%$ \\
\hline Burguesia & 6 & 25,0 & 18 & 75,0 & 24 & 100,0 \\
\hline Peq. Burguesia & 30 & 30,6 & 68 & 69,4 & 98 & 100,0 \\
\hline Prolet. + Sub. & 134 & 50,8 & 129 & 49,2 & 262 & 100,0 \\
\hline TOTAL & 170 & 44,2 & 215 & 55,8 & $385^{*}$ & 100,0 \\
\hline \multicolumn{7}{|c|}{ (") Em três casos o parto foi à fórceps } \\
\hline $\begin{array}{l}X^{2} \text { calculado }=1 \\
X^{2} \text { critico }=5,99 \\
\left({ }^{* *}\right)=\text { Nivel de si }\end{array}$ & $\begin{array}{l}4^{* * *} \\
\text { ícân de } \\
\text { ícâne }\end{array}$ & $\begin{array}{l}\text { erdad } \\
=5 \%\end{array}$ & & & & \\
\hline
\end{tabular}

\section{Tabela 4 - DISTRIBUIÇÃO DAS CRIANÇAS SEGUNDO A RENDA FAMILIAR PER CAPITA E O TIPO DE PARTO, LONDRINA, 1990}

\begin{tabular}{|c|c|c|c|c|c|c|}
\hline \multirow{3}{*}{$\begin{array}{l}\text { Renda per } \\
\text { capita }\end{array}$} & \multicolumn{4}{|c|}{ Tipo de parto } & \multirow{2}{*}{\multicolumn{2}{|c|}{ TOTAL }} \\
\hline & \multicolumn{2}{|c|}{ Normal } & \multicolumn{2}{|c|}{ Cesárea } & & \\
\hline & $\mathbf{N}$ & $\%$ & $N$ & $\%$ & $\mathbf{N}$ & $\%$ \\
\hline $0:-1$ & 94 & 55,0 & 77 & 45,0 & 171 & 100,0 \\
\hline $1 /-2$ & 44 & 36,4 & 77 & 63,6 & 121 & 100,0 \\
\hline $2 /$ & 32 & 34,4 & 61 & 65,6 & 93 & 100,0 \\
\hline TOTAL & 170 & 44,2 & 215 & 55,8 & $385^{\star}$ & 100,0 \\
\hline
\end{tabular}

(`) Em trēs casos o parto foi à fórceps

$\mathrm{X}^{2}$ calculado $=14,67^{\star *}$

$X^{2}$ crítico $=5,99$ (grau de liberdade $=2$ )

$\left({ }^{* *}\right)=$ Nivel de signifícáncia $=5 \%$

\subsection{3 - Puericultura}

A concentração alcançada pela assistência de puericultura, entre as crianças da amostra maiores de 12 meses, foi de 10,8 consultas em média no primeiro ano de vida, variando segundo a escolaridade do chefe de família entre 7,7 consultas entre os analfabetos e 13,1 no nivel colegial e superior. Verificou-se também que a assistência foi iniciada no primeiro mês de vida em $80 \%$ das crianças.

Os resultados do local onde foi realizada a puericultura, evidenciaram que a rede básica de Postos de Saúde e o ex-PAM do INAMPS, somados ao ambulatório do HU foram responsáveis por $64 \%$, ficando os serviços privados com apenas $23 \%$ das familias estudadas; e apenas $6 \%$ das mães procuraram a puericultura em outra cidade e $7 \%$ não a realizaram no primeiro ano de vida.

\subsection{4 - Vacinação}

Do total de 388 crianças da amostra, foram analisadas as 247 que apresentavam idade entre $12 \mathrm{e}$ 59 meses e cartão de vacina, tendo sido excluídas na análise as 48 crianças nesta faixa etária que năo apresentaram o cartão. Em relação ao BCG intradérmico, observou-se a presença de cicatriz vacinal em $95 \%$ das crianças e o cartão atestava a vacinação em $98,4 \%$; Sabin, três doses $(97,6 \%)$, DPT, três doses $(97,6 \%)$ e Anti-Sarampo, uma dose $(98,0 \%)$.

Observou-se que as variáveis estudadas não influenciaram a vacinação, não havendo associação significativa com renda per-capita, escolaridade ou classe social. O que revelou proporção muito satisfatória de crianças adequadamente vacinadas, acima de $97 \%$ para todas as vacinas (Tabela 5 e Gráfico 2).

\section{4-DISCUSSÃO}

Acredita-se que a amostra represente bem 0 universo de crianças menores de cinco anos do município de Londrina, porque não se obtiveram dados de mais do que uma criança por domicílio. 
Tabela 5 - DISTRIBUIÇÃO DAS CRIANÇAS ESTUDADAS (“) SEGUNDO O CUMPRIMENTO DO ESQUEMA DE IMUNIZAÇÃO EAS CLASSES SOCIAIS, NA ZONA URBANA DO MUNICIPIO DE LONDRINA, 1990

\begin{tabular}{|c|c|c|c|c|c|c|c|c|c|c|}
\hline \multirow{2}{*}{$\begin{array}{l}\text { Classe } \\
\text { Social }\end{array}$} & \multirow{2}{*}{$\begin{array}{l}\text { Sem cartão } \\
N\end{array}$} & \multirow{2}{*}{$\begin{array}{l}\text { BCG } \\
\text { n. }\end{array}$} & \multirow[b]{2}{*}{$\%$} & \multicolumn{2}{|c|}{ SABIN } & \multicolumn{2}{|l|}{ DPT } & \multicolumn{2}{|c|}{ Anti-Sarampo } & \multirow{2}{*}{$\begin{array}{l}\text { Com Cartão } \\
\mathrm{N}_{2}\end{array}$} \\
\hline & & & & n. & $\%$ & $\mathrm{n}$ & $\%$ & n. & $\%$ & \\
\hline Burguesia & 5 & 12 & 100,0 & 11 & 91,7 & 11 & 91,7 & 12 & 100,0 & 12 \\
\hline Pequena Burguesia & 11 & 60 & 95,2 & 60 & 95,2 & 60 & 95,2 & 60 & 95,2 & 63 \\
\hline Proletariado + Sub & 32 & 171 & 99,4 & 170 & 98,8 & 170 & 98,8 & 170 & 98,8 & 172 \\
\hline TOTAL & 48 & 243 & 98,4 & 241 & 97,6 & 241 & 97,6 & 242 & 98,0 & $247^{* *}$ \\
\hline \multicolumn{4}{|c|}{$\begin{array}{l}x^{2} \text { calculado }=0,082^{*} \\
x^{2} \text { critico }=12,6 \text { (grau de liberdade }=6 \text { ) }\end{array}$} & \multicolumn{7}{|c|}{$\begin{array}{l}N_{1}=\text { excluidas da análise } \\
N_{2}=\text { analisadas } \\
(*) \text { Crianças com idade entre } 12 \text { e } 59 \text { meses }\end{array}$} \\
\hline
\end{tabular}

\section{GRÁFICO 2-COBERTURA VACINAL DAS GRIANÇAS ESTUDADAS SEGUNDO A ESCOLARIDADE DO CHEFE DE FAMIUA NAZONA URBANA DO MUNICIPIO DE LONDRINA, 1990}

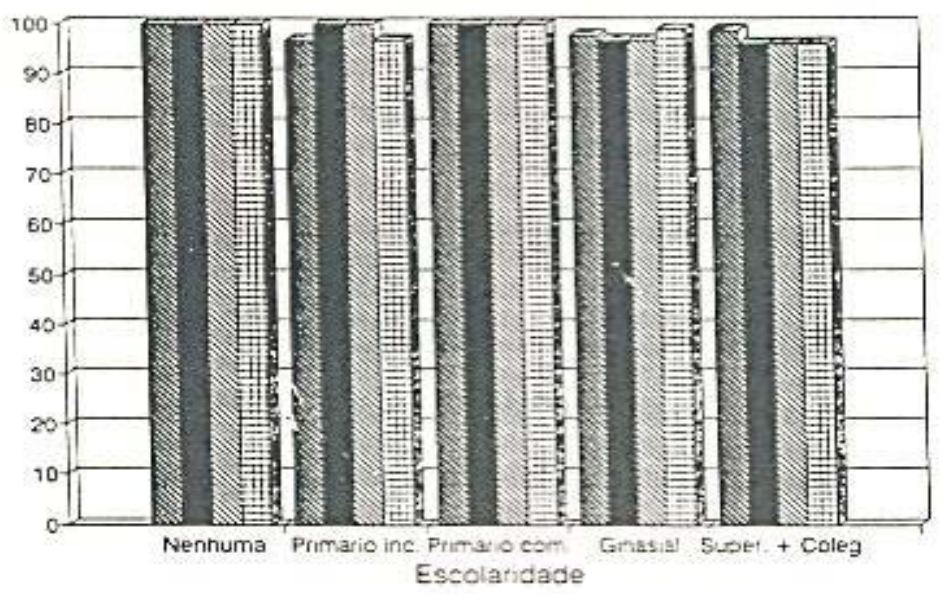

BCG BCABIN SA DPT

Com relação ao número médio de pessoas por domicilio, encontramos 4,5 ; porém, é possível que a seleção de familias com crianças menores de cinco anos esteja levando a este resultado; porque quando se compara com os dados preliminares do Censos de 91 , - município de Londrina tem uma média de 3,9 habitantes por domicílio (IBGE, 1992).

Com relação ao rendimento médio mensal das familias entrevistadas, quando comparada com as informaçōes obtidas pelo Censo de 1980 (IBGE, 1984). verificou-se que a distribuiçăo da população por faixa de renda não apresentou diferença, indicando a adequada seleçăo dos casos para estudo.

Comparando a distribuição da escolaridade dos chefes de familia, com estudo semelhante realizado no município de São Paulo (MONTEIRO, 1988), verificouse que em Londrina eles apresentaram melhores niveis de escolaridade.

Comparando os resultados encontrados por BARROS (1983), em estudo de morbidade e consumo de medicamentos em relação à saúde e classe social, realizado em Ribeirão Preto, verificou-se que existe uma semelhança nas proporçōes das classes, sendo o proletariado o mais freqüente, seguido pela pequena burguesia e burguesia; com a pequena burguesia mais numerosa neste trabalho.

SINGER (citado por BARROS, 1983) refere:

-Na verdade, apenas os autônomos proprietários pertencem à pequena burguesia. Os autônomos destituídos de qualquer propriedade são antes vendedores de força de trabalho que não encontram compradores para ela no mercado de trabalho. Constituem, nesse sentido, parte do exército industrial de reserva e, como tal, participam do subproletariado".

Concordando com esta análise, os autônomos sem negócio, foram incorporados ao subproletariado, mesmo assim a pequena burguesia londrinense representou aproximadamente $25 \%$ das classes sociais.

Pode-se observar que a assistência básica à saúde materno-infantil no município de Londrina apresentou grande cobertura, atingindo inclusive os estratos de mais baixo nivel sócio-econômico.

Comparando essas taxas de coberturas da assistência pré-natal e ao parto observadas nesse estudo, com as registradas pelo IBGE (1980) para outras áreas urbanas do pais e as encontradas em inquérito realizado em São Paulo (MONTEIRO, 1988), verificouse que a situação de Londrina é superior à apontada para o conjunto das áreas umanas do Brasil, sendo 
comparável à encontrada em São Paulo, destacandose a homogeneidade da distribuição da assistência.

No caso da assistência ao parto, não se pode afastar a possibilidade de que determinados números encontrados neste trabalho estejam superestimados, porque, além de óbitos infantis e pré-escolares, devese considerar também a ausência de natimortos. Importante destacar que todas as estimativas deste estudo foram centradas em crianças existentes em determinado momento, não sendo aplicáveis, portanto, às gestações que não evoluíram a nascidos vivos e também às crianças que morreram antes de completar os cinco anos de idade. Não se pode deixar de considerar que, nestes casos, a assistência prestada talvez tenha sido pior do que a oferecida para os sobreviventes.

Com relação à puericultura, não se deve esperar que este fato tenha ocorrido com muita intensidade, porque não foram consideradas, apenas as crianças que morreram antes dos cinco anos de idade. Entre 1979 e 1990 a mortalidade infantil de Londrina apresentou uma redução de $43 \%$, estando em 19,14 por mil nascidos vivos em 1992. No Paraná este coeficiente está em tomo de 30 por mil e no Brasil, acima de 50 (BIS, 1994).

A Tabela 6 compara indicadores de cobertura de assistência materno-infantil encontrados em nosso estudo com os obtidos no município de São Paulo (MONTEIRO, 1988). A comparação sugere que a assistência pré-natal em Londrina é melhor que a encontrada em São Paulo, podendo esta ser considerada muito satisfatória. A assistência hospitalar ao parto pode ser considerada praticamente universal nas duas cidades. Sobre a cobertura vacinal, os dados encontrados são ainda mais expressivos, atingindo a 'quase totalidade das crianças na faixa etária estudada.

\section{Tabela 6 - ESTIMATIVA DE INDICADORES DA COBERTURA DA ASSISTÊNCIA MATERNO- INFANTIL DO MUNICÍPIO DE SÃO PAULO EM INQUÉRITO POPULACIONAL REALIZADO EM 1984/85, COMPARADO COM LONDRINA, 1990}

Assistência pré-natal

$\%$ cobertura

Assistência ao parto

$\%$ partos hospitalares

Assistência de puericultura

$\%$ crianças vacinadas

$\begin{array}{ll}\text { São Paulo * } & \text { Londrina ** } \\ 92,9 & 98,4\end{array}$

99,0

$\begin{array}{lcc}\text { BCG } & 89,0 & 98,4 \\ \text { SABIN } & 86,7 & 97,6 \\ \text { DPT } & 85,1 & 97,6 \\ \text { Anti-Sarampo } & 85,9 & 98,0 \\ & \\ \text { * Baseado em amostra de } 393 \text { crianças de } 0 \text { a } 23 \text { meses de idade (assisténcia pré-natal e ao parto) e de } 833 \text { crianças } \\ \text { de } 12 \text { a } 59 \text { meses de idade (vacinação). } \\ \text { * Baseado em amostra de } 388 \text { crianças de } 0 \text { a } 59 \text { meses de idade (assistência pré-natal e ao parto) e de } 260 \\ \text { crianças de } 12 \text { a } 59 \text { meses de idade (vacinação). }\end{array}$

Comparando nossos resultados com os de alguns países sub-desenvolvidos, observamos que apenas a China em 1980 apresentava uma cobertura de atenção pré-natal maior, em média $98 \%$. Para 1983 , enquanto uma parteira diplomada ou um médico assistiam praticamente a todos os nascimentos nos países desenvolvidos - e em 1990 em Londrina -, este requisito só se cumpria em $64 \%$ dos nascimentos da América Latina, $49 \%$ dos da Ásia e em $34 \%$ dos da África (ROYSTON \& ARMSTRONG, 1991).

Quanto à qualidade da assistência matemo-infantil prestada , um aspecto preocupante se refere à elevadíssima proporção de partos cirúrgicos, registrada em um inquérito populacional: $55,8 \%$. A maior proporção de cesarianas foi encontrada nos estratos da população de melhor nível sócio-econômico, que certamente goza de melhores condições de saúde e de melhor acompanhamento pré-natal, o que sugere que não sejam razões essencialmente técnicas as responsáveis pela maioria das indicações cirúrgicas feitas no município. Em quase todos os grandes hospitais dos países em desenvolvimento cerca de $85 \%$ dos partos são normais (ROYSTON \& ARMSTRONG, 1991).

No inquérito realizado em São Paulo em 84/85, a freqüência de nascimentos por cesariana foi de $41 \%$, e para o conjunto das áreas urbanas do país, está estimada em $34 \%$ (IBGE, 1984).

As razões de tal fato, segundo autores que examinaram a fundo a questão, incluiriam interesses financeiros de médicos e a própria pressão da clientela 
que acabou sendo induzida a ver o parto cirúrgico como procedimento nomal e comiqueiro e o parto nomal como algo ulirapassado. Outro aspecto importante prende-se ao fato da ausencia de outras formas alternativas seguras de aniciconcepção, sendo expressiva a proporcăo de mulheres que optam pela laqueadura de trompas; nesmo admitindo-se como complexa. Não se pode deixar de reconhecer, no fato, 0 aspecto negativo no desempenho da assistência materno-infantil prestada à população de Londrina (MICTORA et al., 1988).

A análise da cobentura vacinal na amostra estudada foi feita baseada na metodologia empregada por diversos autores já citados anteriomente, o que nos permite comparå-la com a publicaça Situaça Mundial da Infância (UNICEF, 1992). Nesta publicação, o Brasil situa-se com uma percentagem de crianças com um ano totalmente imunizadas para a ano de $1989-90$ de: Tuberculose 78\%, Sarampo 78\%, DPT 81\% e Pólio $93 \%$. O que representa ser a situação da zona urbana do município de Londrina muito superior à brasileira $e$ comparável à os países que apresentam baira tara de mortalidade de menores de 5 anos por mil nascidos vivos (TMM5) e às maiores cobenuras do mundo. A meta estabelecida para a década de 30, pelo Encontro Mundial de Cúpula pela Crianca, realizado no final de Setembro de 1990, na sede das Naçőes Unidas, de se atingir 90\% de imunizaçäo até o ano 2000, jổ foi atingiola em Londrina em1990, para rodas as vacinas do primeiro ano de vida.

O impacto produzido por esta cobertura na mortalidade pode ser validado, pelos dados de mortalidade por doenças preveniveis por vacina no primeiro ano de vida, apesar de todas as limitaçôes do sistema de informaçóes de mortalidade, como se pode constatar junto à Seçäo de Epidemiologia da $17^{2}$ Regional de Saúde SUS/Londrina. Com relaçăo aos óbitos em crianças menores de cinco anos por Meningite Tuberculosa, registraram-se dois casos em 1989, dois casos em 1990 e um caso em 1991; Difteria desde 1987 não existem sequer casos notificados; Coqueluche houve um óbiro em 1989; Tétano Neonatal desole 1988 não existem casos notificados; Poliomielite desde 1987 não existem casos notificados e a partir de 1990, nem suspeitos; Sarampo registraram-se dois óbitos em 1980 e é provável que esie dzdo esieja subestimado.

\section{CONCLUSÖES}

Foi analisada a coberura da assistência prẻ-natal, 2o parto, à puericultura e ə̀ vacinação, segundo as condições sócioueconomicas descrevendo-se as condiçöes da moradia e saneamento básico, no período de julho ఏ̀ dezembro de 1990, na cidade de Londrina. Para isso, urilizoumse a matodologia do estudo transversal, sendo a amosira estudada de 388 crianças menores de 5 anos.

Comparando-Se os resultados dos inquéritos populacionais de oûras áreas urbanas do país, que avaliaram elementos da qualidade da assistência matemo-iniantil, com os resultados obtidos na zona urbana do municipio de Londrina, a diferença principal é que nå fol evidenciada desigualdade marcante na assistência prestada às criancas dos dî̉erentes estratos sociais da populaça.

A2 and́lise das variáveis: renda, escolaridade do chefe de ramilia e classes sociais, verificou-se que:

- Foi observada diferenca estatisticamente significativa quanto à distribuiçăo da renda en faizas de salário minimo per-capita mensais, quando comparada com a escolaridade.

- A distribuiça da escolaridade do chefe de framilia - da mäe foram semelhantes, apresentando baizo percentual de analiabeios $(3,3 \%)$, a ali o perceniual de individuos que cursaram o ginasial (respectivamente $36 \%$ - 37\%), nă havendo direrenç estauisticanente signoficativa.

- Na distribuică am clssses sociais, foi ohsenso: uma associaga significativa tanto em reiaço à renda per-capils, como em relaça @ escolaridade do chefe de iamill:, o que reforça a validade da utilizaço do conceito de classe social em estudos epidemióógicos, principalmente em estudos populacionais.

A avaliacão da assistência \&े saúde matemo invianil, revelou a disponibilidade de una rede de senvicos de atença primária no municipio que desenvolve as programacôs de pré.nata! e puericufura com ampla cobertura populacional. A assistência ao parto foi hospî̉aler em 99,7\% das mutheres.

- O programa de pré-natal foi iniciado no primeiro uninestre por $82 \%$ des mulheres entrevistadas, näo houve associaça significante entre o periodo de inicio do pré. natal e renda pep-capita, escolanidade ou classe social.

- A análise das modalidades de parto mostrou associaço signifiçîti e inversamente proporcional com a renda per-capita, escolaridede do chefe de familia. classes sociais e proporcåo de pano nomal.

O programa de puericultura apresentou uma média de 10,8 consulias no primeiro ano de vióa. anire as crianças maiores de 12 meses, sendo menor $(7,7)$ entre as criancas ors familias analfaberas, o que ainda है uma realidade médicosanî́ria em nosso meio.

- Na snálise dia vacinaça,o, observou-se gue, no cumprimento do esquema de imunimaça, näo existiu diferenca significativa entre os offerentes niveis de rends per-capita, escolaridade do chefe de immilia ou classe social, sendo a coberina bastante alia para todas as vacinas.

Obsenou-se que a ong anicaca dos senicos de saúde local nå foi apenas uma experiência em processo, mas principalmente um fator que muito connibuiu para consolidaço de una rede básica de saúde, viabilizando uma ampla assistência matemoinfanil em Lonodrina.

Finalizando, acredite-se que o processo de municipalizaço das açes de saúde, que vêm sendo implementades desde 1970, necessita de un passo frente, a run de que nåo só mories sejam cvitacias, mas que um novo nivel de compromisso de atendimenio seja estabelecio com toda a sociedade, no sentio da universatiza com qualidade. 
UNIVERSIDADE ESTADUAL DE LONDRINA

CENTRO DE CIÊNCIAS DA SAÚDE - SETOR DE SAÚDE COMUNITÁRIA

NESCO/NÚCLEO DE ESTUDOS DE SAÚDE COLETHYA

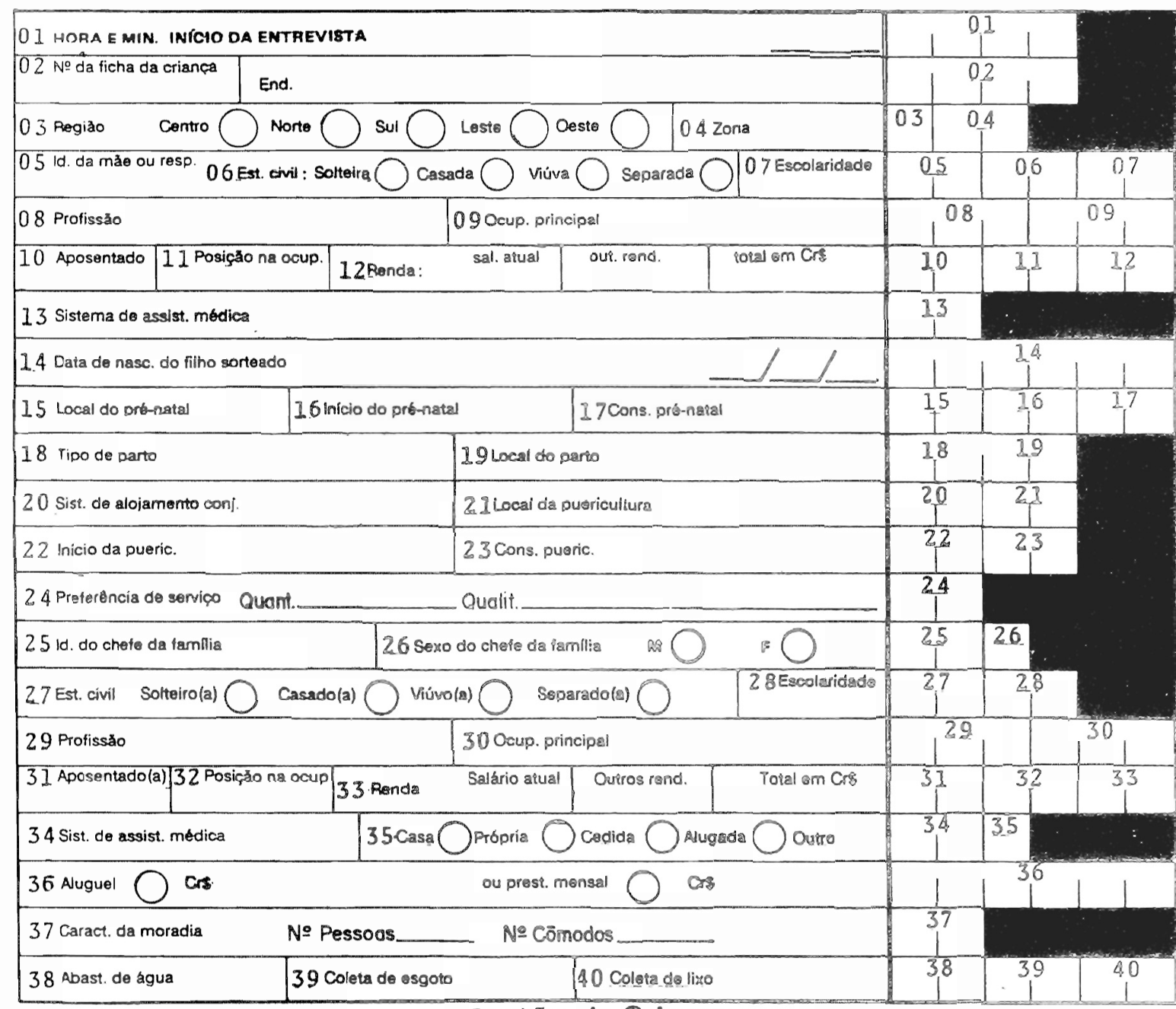

Figura 1 - Formulário 
CAMPOS, João José Batista de; FORSTER, Aldaisa Cassanho; SOARES, Darli Antônio. Study on health conditions of children in the urban area of Londrina. Semina: Ci. Biol./Saúde, Londrina, v. 16, n. 2, p. 224-233, Jun. 1995.

ABSTRACT: This study was undertaken in order to analyse infant's health conditions in the urban area of Londrina aiming to contribute to the evaluation of available Health Services. This investigation was a transversal study on sanitation, socio-economical status health system and the environment. The studied sample included 388 children from days to 59 months, from all five urban areas of Londrina. Three variables were selected: educational level of the head of the family, social class and monthly income per person. The head of the family presented a high level of education and a direct correlation between education, monthly income per person and social status was found. The results showed an extremely high percentage of ceasarian section $(55,8 \%), 79,4 \%$ of children were registered in the Well Baby Clinic during the first month of life with an average of 10,8 visits per child during the first year. In relation to the immunization schedule it was found that $98,4 \%$ were immunised against tuberculosis with BCG (one dose); $97,6 \%$ received three doses against poliomyelitis (Sabin); $97,6 \%$ were given three doses of DPT and $98,0 \%$ received one dose against measles. The results showed no statistical difference for children over one year old. Based on the resulfs the author concluded that maternal and child health senvices in the urloan area of Londrina are extensive and of very good quality.

KEY-WORDS: Children health, Valuation of health senvices, Epidemiology suncey.

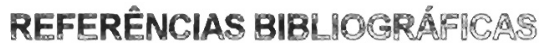

ALMEIDA, M. J. A organizaçăo de serviços do saúde a nivel local: Registros de uma experiência em processo. Rio de Janeiro, 1979. Dissertação (Mestrado) - Instituic de Medicina Social - Universidade Estadual do Rio de Janeiro, 4979

BARROS, F. C.; VICTORA, C. G. Epidemiologia da saúde infantil: um manual para diagnósticos comunitários. \$ão Paulo: Hucitec, 1991.

BARROS, M. B. A. Saúde e Classe Social: um estudo sobre Morbidade e Consumo de Medicamentos, São Paulo, 1983. Tese (Doutorado) - Faculdade de Medicina de Ribeirão Preto - Universidade de São Paulo, 1983.

BIS (Boletim Informativo da Saúde) - A mortalidado iniontil em Londrina. Londrina: Secretaria de Saúde, 9. 1994.

CARVALHEIRO, J. R. Levantamento de Condiçőes de Saúde por Entrevistas Domiciliares. São Paulo, 1975. Tese (Livpe。 Docência) - Faculdade de Medicina de Ribeirão Preto o Universidade de São Paulo, 1975.

CORDON!, Lo Serviços Municipais de Saúde no Paraná. Tendencias e Perspectivas. São Paulo, 1987. Tese (Doutoramento) - Faculdade de Saúde Pública Universidade de São Paulo, 1987.

FORSTER, A C. Cobertura dos serviços de imunização de Ribeirão Preto através de entrevistas domiciliárias. São Paulo, 1979. Dissertação (Mestrado) - Faculdade de Medicina de Ribeirāo Preto - Universidade de São Paulo, 1979.

FORSTER, $\mathbb{A}_{\text {e }}$. Hospitalizaçס̋es a classes sociais Sảo Paulo,
1984. Trse (Dourerado) - Faculdade de Medicina de

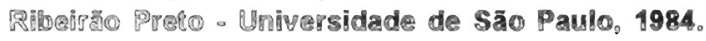

1BGE (Bnstîture Brasileiro de Geografia E Estatistica) - Ponfil จ จ

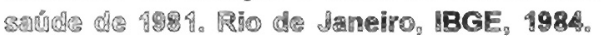

ABCE (instituto Brasileiro de Geografia e Estatística) 。 Sinopse Preliminar do Censo Demográfico de 1991. Rio de daneiro, 18GE, 4992.

1TO, A. W. H. Estado nutricional $\theta$ hábito alimentar do escolares no municipio de Londrina/PR. Londrina, 9984.

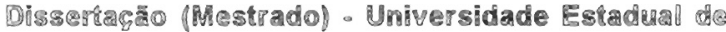

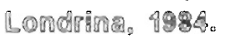

MORTERRO, C. A. Saúdo a nutriça das crianças do Säo Paulo.

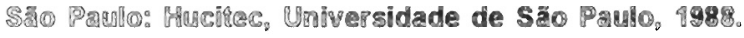

MOSER, C. A. Survey Methods in Social Investigation. Londorn: Heinemann Educational Books, 1958.

ROYSTON, E. ARMSTRONG, S. Prevencion de la Mortalidad Matema. Ginebra: Organización Mundial de 8. Salud.

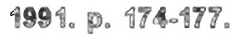

UNICEF (Fundo das Nações Unidas para a infância) Situação Mundial da Iníancia. Brasília: Unicef, 1992.

UNIVERSIDADE ESTADUAL DE LONDRINA. PROJETO UNI Londrina uma nov iniciativa na educação dos profissionais de saúde: Uniżo com a comunidade (PROUNALQ). Londrina, 4992.

MCTORa, C. G. et at. Epidemiologia da desigualdade. São Parto: Hucitec, 1988. 